\title{
On the Darboux transformations and sequences of p-orthogonal polynomials
}

\author{
D. Barrios Rolanía, J.C. García-Ardila, D. Manrique \\ Dpto. de Matemática Aplicada a la Ingeniería Industrial \\ Dpto. de Inteligencia Artificial \\ Universidad Politécnica de Madrid
}

\begin{abstract}
For a fixed $p \in \mathbb{N}$, sequences of polynomials $\left\{P_{n}\right\}, n \in \mathbb{N}$, defined by a $(p+2)$ term recurrence relation are related to several topics in Approximation Theory. A $(p+2)$-banded matrix $J$ determines the coefficients of the recurrence relation of any of such sequences of polynomials. The connection between these polynomials and the concept of orthogonality has been already established through a $p$-dimension vector of functionals. This work goes further in this topic by analyzing the relation between such vectors for the set of sequences $\left\{P_{n}^{(j)}\right\}, n \in N$, associated with the Darboux transformations $J^{(j)}, j=1, \ldots, p$, of a given $(p+2)$-banded matrix $J$.
\end{abstract}

\section{Introduction}

For a fixed $p \in \mathbb{N}$ we consider a sequence of polynomials $\left\{P_{n}\right\}, n \in \mathbb{N}$, defined by a $(p+2)$-term recurrence relation

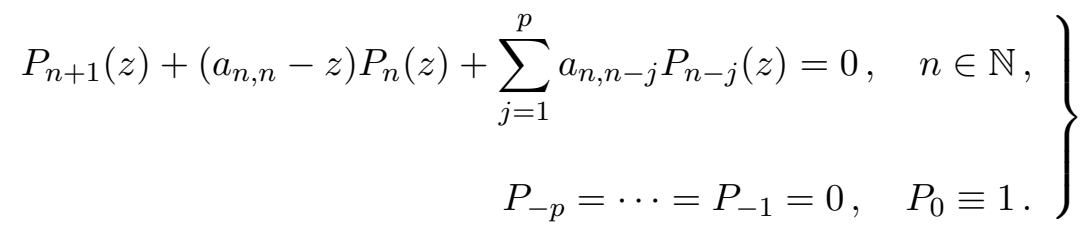

These polynomials are related with several topics such as Hermite-Padé approximants and vector continued fractions ( [5, 9]). In particular, this kind of polynomials plays an essential role in the study of some integrable systems (see for instance [1-3]).

The relation between the concept of orthogonality and the sequences of polynomials verifying a $(p+2)$-term recurrence relation was established in [13] in the following wellknown result.

Lemma 1 With the above notation, the following statements are equivalent.

(i) $\left\{P_{n}\right\}, n \in \mathbb{N}$, verify (1) with $a_{n, n-p} \neq 0$ for all $n \in \mathbb{N}$.

(ii) There exists a vector of functionals $\nu=\left(\nu_{1}, \ldots, \nu_{p}\right)$, where each $\nu_{r} \in \mathcal{P}^{\prime}, r=$ $1, \ldots, p$, is defined on the space of polynomials $\mathcal{P}$ verifying

$$
\left\{\begin{array}{l}
\nu_{r}\left[z^{k} P_{n}(z)\right]=0, \quad k=0,1, \ldots, \quad k p+r \leq n, \quad n \in \mathbb{N}, \\
\nu_{r}\left[z^{k} P_{k p+r-1}(z)\right] \neq 0, \quad k=0,1, \ldots
\end{array}\right.
$$


In the sequel, we call vector of p-orthogonality to any vector of functionals $\nu=$ $\left(\nu_{1}, \ldots, \nu_{p}\right)$ verifying (2). In this case, we say that $\left\{P_{n}\right\}$ is a sequence of p-orthogonal polynomials with respect to $\nu$.

For a sequence of polynomials $\left\{P_{n}\right\}$ defined in (10), the sequence of linear functionals $\left\{\mathcal{L}_{n}\right\}, n=0,1, \ldots$, given by

$$
\mathcal{L}_{j}\left[P_{i}\right]=\delta_{i, j}, \quad i, j=0,1, \ldots,
$$

plays an relevant role in the study of the orthogonality. $\left\{\mathcal{L}_{n}\right\}$ is called dual sequence, and it is the unique sequence of functionals verifying (3). It is easy to check that the $p$ first terms $\mathcal{L}_{r}, r=0,1 \ldots, p-1$, of the dual sequence verify the orthogonality conditions (2). This fact proves the existence of some vector of $p$-orthogonality associated with each arbitrary sequence $\left\{P_{n}\right\}$ of polynomials. However, the uniqueness of a vector functional as in (2) is not guaranteed. In fact, P. Maroni characterized in [10] these vectors of $p$-orthogonality as $\left(\nu_{1}, \ldots, \nu_{p}\right)$ such that

$$
\begin{aligned}
\nu_{1} & =\lambda_{1,0} \mathcal{L}_{0} \\
\nu_{2} & =\lambda_{2,0} \mathcal{L}_{0}+\lambda_{2,1} \mathcal{L}_{1} \\
& \vdots \\
\nu_{p} & =\lambda_{p, 0} \mathcal{L}_{0}+\lambda_{p, 1} \mathcal{L}_{1}+\cdots+\lambda_{p, p-1} \mathcal{L}_{p-1}
\end{aligned}
$$

being $\lambda_{i, j} \in \mathbb{C}$ and $\lambda_{i, i-1} \neq 0$ for $i, j+1 \in\{1, \ldots, p\}$.

Associated with (10), it is possible to define the $(p+2)$-banded matrix $J$ whose entries are the coefficients of the recurrence relation,

$$
J=\left(\begin{array}{cccccc}
a_{0,0} & 1 & & & & \\
a_{1,0} & a_{1,1} & 1 & & & \\
\vdots & \vdots & \ddots & \ddots & & \\
a_{p, 0} & a_{p, 1} & \cdots & a_{p, p} & 1 & \\
0 & a_{p+1,1} & & \ddots & \ddots & \ddots \\
& 0 & \ddots & & & \\
& & \ddots & & &
\end{array}\right),
$$

where we assume $a_{j+p, j} \neq 0, j=0,1, \ldots$

The use of discrete Darboux transformations was proposed in [11,12] with the focus in the application to the Toda lattices. In [1 6] this study was extended to $(p+2)$ banded matrices as (5). In the present work we are concerned about finding relations between the vectors of $p$-orthogonality associated with the Darboux transformations of such matrices (5). Thereby this paper complements the analysis that has been done in [4] for the Geronimus transformations. We include here the following summary, with the more relevant concepts, for an independent reading.

Let $C \in \mathbb{C}$ be such that the main determinants of the infinite matrix $J-C I$ verify

$$
\operatorname{det}\left(C I_{n}-J_{n}\right) \neq 0 \text { for each } n \in \mathbb{N}
$$

Due to the well-known fact that $P_{n}(z)=\operatorname{det}\left(z I_{n}-J_{n}\right)$, (6) is equivalent to $C \in \mathbb{C}$ is not a zero of the sequence $\left\{P_{n}\right\}$. (Here and in the sequel, given a semi-infinite matrix $A$, 
we denote by $A_{n}, n \in \mathbb{N}$, the finite matrix of order $n$ formed with the first $n$ rows and columns of $A$.) In these conditions, there exist two lower and upper triangular matrices, $L$ and $U$ respectively,

$$
L=\left(\begin{array}{ccccc}
1 & & & & \\
l_{1,1} & 1 & & & \\
\vdots & \ddots & \ddots & & \\
l_{p, 1} & l_{p, 2} & \ldots & 1 & \\
0 & l_{p+1,2} & \ddots & & \ddots \\
& 0 & \ddots & \ddots & \ddots
\end{array}\right)
$$

and

$$
U=\left(\begin{array}{cccc}
\gamma_{1} & 1 & & \\
& \gamma_{p+2} & 1 & \\
& & \gamma_{2 p+3} & \ddots \\
& & & \ddots
\end{array}\right),
$$

such that

$$
J-C I=L U
$$

is the unique factorization of $J-C I$ in these conditions (see [8] for details). In the sequel we assume $C \in \mathbb{C}$ fixed.

In [6] the following factorization of $L$ was given.

Lemma 2 [6], Theorem 1, pp. 118] In the above conditions, if $l_{p+i, i+1} \neq 0$ for each $i=0,1, \ldots$, then there exist $p$ bi-diagonal matrices

$$
L^{(j)}=\left(\begin{array}{cccc}
1 & & & \\
\gamma_{j+1} & 1 & & \\
& \gamma_{p+j+2} & 1 & \\
& & \gamma_{2 p+j+3} & \ddots \\
& & & \ddots
\end{array}\right), j=1,2, \ldots, p
$$

with $\gamma_{(k-1) p+j+k} \neq 0$ for all $k \in \mathbb{N}$, verifying

$$
L=L^{(1)} L^{(2)} \cdots L^{(p)}
$$

Moreover, for each $j \in\{1,2, \ldots, p-1\}$ it is possible to choose certain set of $p-j$ elements $\gamma_{j+1}, \gamma_{p+j+2}, \ldots, \gamma_{(p-j) p}$ of $L^{(j)}$ such that the factorization (9) is unique for the fixed set of $p(p-1) / 2$ points

$$
\left.\begin{array}{ccccc}
\gamma_{2}, & \gamma_{p+3} & \cdots & \cdots & \gamma_{(p-1) p} \\
\gamma_{3}, & \gamma_{p+4} & \cdots & \gamma_{(p-2) p}, & \\
\vdots & \vdots & \cdots & & \\
\gamma_{p-1}, & \gamma_{2 p}, & & & \\
\gamma_{p} & & &
\end{array}\right\}
$$


(77) and (9) provide the so called Darboux factorization of $J-C I$, defined as

$$
J-C I=L^{(1)} L^{(2)} \cdots L^{(p)} U,
$$

where $L^{(1)}, L^{(2)}, \ldots L^{(p)}$ are bidiagonal matrices as in (8) given in Lemma 2 for certain set (10) of $p(p-1) / 2$ fixed entries. Each circular permutation of a Darboux factorization gives a new $(p+2)$-banded matrix,

$$
J^{(j)}=C I+L^{(j+1)} L^{(j+2)} \cdots L^{(p)} U L^{(1)} L^{(2)} \cdots L^{(j)}, j=1,2, \ldots, p .
$$

Definition 1 The permutations $J^{(j)}$ given in (12) are called Darboux transformation of $J-C I$.

As a consequence of the Darboux transformations, for each $j \in\{1, \ldots, p\}$ it is possible to define a new sequence of polynomials $\left\{P_{n}^{(j)}\right\}$ verifying a $(p+2)$ recurrence relation,

$$
\left.\begin{array}{r}
P_{n+1}^{(j)}(z)+\left(a_{n, n}^{(j)}-z\right) P_{n}^{(j)}(z)+\sum_{s=1}^{p} a_{n, n-s}^{(j)} P_{n-s}^{(j)}(z)=0, \quad n \in \mathbb{N}, \\
P_{-p}^{(j)}=\cdots=P_{-1}^{(j)}=0, \quad P_{0}^{(j)} \equiv 1 .
\end{array}\right\}
$$

Another way to write (13) is

$$
\left(J^{(j)}-z I\right) v^{(j)}=0
$$

where

$$
v^{(j)}(z)=\left(P_{0}^{(j)}(z), P_{1}^{(j)}(z), \ldots\right)^{T}, \quad j=0,1, \ldots, p .
$$

Here and in the following, we extend the notation taking $P_{n}^{(0)}=P_{n}, n \in \mathbb{N}$ and $J^{(0)}=J$.

Since Lemma 1, in the above conditions there exists some vector of $p$-orthogonality

$$
\nu^{(j)}=\left(\nu_{1}^{(j)}, \ldots, \nu_{p}^{(j)}\right), \quad j=1, \ldots, p,
$$

such that the corresponding conditions of orthogonality like (2) are verified.

In this work we analyze some relations between the vectors of $p$-orthogonality $\nu=$ $\left(\nu_{1}, \ldots, \nu_{p}\right)$ associated to the polynomials $\left\{P_{n}\right\}$ and the vectors of $p$-orthogonality (15), $j=1, \ldots, p$, for a Darboux factorization of $J-C I$ under some conditions.

Definition 2 Here and in what follows, $(z-C) \nu_{i}, i=1, \ldots, p$, is a linear functional defined on the space $\mathcal{P}[z]$ of polynomials as

$$
(z-C) \nu_{i}[q]=\nu_{i}[(z-C) q]
$$

for each $q \in \mathcal{P}[z]$.

We recall that it is possible to have several vectors of $p$-orthogonality associated with each sequence of polynomials verifying a $(p+2)$-term recurrence relation. If $\left(\nu_{1}, \ldots, \nu_{p}\right)$ 
verifies (2) then for $\left(c_{1}, \ldots, c_{p}\right) \in \mathbb{C}$ also $\left(c_{1} \nu_{1}, \ldots, c_{p} \nu_{p}\right)$ verifies (2). In a more general procedure, the coefficients $\lambda_{i, j}$ of (4) and the determinants

$$
\Delta_{m}^{(j)}=\left|\begin{array}{ccccccc}
\lambda_{j+2,0} & \lambda_{j+3,0} & \cdots & \cdots & \lambda_{m, 0} & \cdots & \lambda_{j+m+1,0} \\
\vdots & \vdots & & & \vdots & & \\
\lambda_{j+2, j+1} & \lambda_{j+3, j+1} & \cdots & \cdots & \lambda_{m, 1} & \cdots & \lambda_{j+m+1, j+1} \\
0 & \lambda_{j+3, j+2} & & & \vdots & & \vdots \\
\vdots & 0 & \ddots & & & & \\
\vdots & \vdots & \ddots & \ddots & \vdots & & \vdots \\
0 & 0 & \cdots & 0 & \lambda_{m, m-1} & \cdots & \lambda_{j+m+1, m-1}
\end{array}\right|,
$$

$j=0,1, \ldots, p-1, m=1,2, \ldots, p-j-1$, play an important role in this paper. Our main contribution is the following.

Theorem 1 Let $\nu=\left(\nu_{1}, \ldots, \nu_{p}\right)$ be a vector of $p$-orthogonality for $\left\{P_{n}\right\}, n \in \mathbb{N}$, as in (4) such that

$$
\Delta_{m}^{(j)} \neq 0, \quad j=0, \ldots, p-1, \quad m=1, \ldots, p-j-1 .
$$

Then there exists a Darboux factorization (11) of $J-C I$ such that, for each $j=1,2, \ldots, p$,

$$
\nu^{(j)}=\left(\nu_{j+1}, \ldots, \nu_{p},(z-C) \nu_{1}, \ldots,(z-C) \nu_{j}\right)
$$

is a vector of p-orthogonality for the sequence of polynomials $\left\{P_{n}^{(j)}\right\}, n \in \mathbb{N}$, associated with the Darboux transformation $J^{(j)}$ of $J-C I$ given in (12) (where we understand $\nu^{(p)}=\left((z-C) \nu_{1}, \ldots,(z-C) \nu_{p}\right)$ in (17) $)$.

Definition 3 In the conditions of Theorem 1 we say that the vectors of p-orthogonality (17) are the Darboux transformations of $\nu$.

In Section 2 some auxiliary results are established. In particular, some connections between the sequences $\left\{P_{n}^{(j)}\right\}, j=0, \ldots, p$, are given for each fixed Darboux factorization. Finally, Theorem 1 is proved in Section 3 .

\section{Orthogonality and Darboux transformations}

In this section we assume that (11) is a fixed Darboux factorization of $J-C I$ corresponding with a given set of entries (10) of the matrices $L^{(1)}, \ldots, L^{(p)}$.

The following result establishes some relationships between the various sequences of polynomials associated with the Darboux transformations of $J-C I$.

Theorem 2 We have the following relations between the sequences of polynomials $\left\{P_{n}^{(j)}\right\}, j=$ $0,1, \ldots, p$.

$$
\begin{aligned}
P_{n}^{(j+1)}(z) & =\frac{P_{n+1}^{(j)}(z)+\sum_{s=0}^{p-1} g_{n+1, n-s+1}^{(j)} P_{n-s}^{(j)}(z)}{z-C}, \quad j=0,1, \ldots, p-1, \quad n \geq 0, \\
P_{n}^{(p)}(z) & =\frac{P_{n+1}(z)-\frac{P_{n+1}(C)}{P_{n}(C)} P_{n}(z)}{z-C}, \quad n \geq 0,
\end{aligned}
$$


where $g_{n+1, n-s+1}^{(j)} \in \mathbb{C}$ for $j=0,1, \ldots, p-1, n \geq 0$ and $s=0, \ldots, p-1$.

Proof.- In [6] was proved that

$$
L^{(j+1)} L^{(j+2)} \cdots L^{(i)} v^{(i)}(z)=v^{(j)}(z), \quad 0 \leq j<i \leq p,
$$

where the product of the triangular matrix $L^{(j+1)} L^{(j+2)} \cdots L^{(i)}$ times the vector $v^{(i)}(z)$ is understanding in a formal sense.

Moreover, from this, (12) and (14),

$$
L^{(j+1)} L^{(j+2)} \cdots L^{(p)} U L^{(1)} \cdots L^{(j)} v^{(j)}(z)=(z-C) v^{(j)}(z), \quad j=0,1, \ldots, p,
$$

where we understand

$$
U L v^{(p)}(z)=(z-C) v^{(p)}(z)
$$

when $j=p$. Replacing $j$ by $j+1$ in (21),

$L^{(j+2)} L^{(j+3)} \cdots L^{(p)} U L^{(1)} \cdots L^{(j)} L^{(j+1)} v^{(j+1)}(z)=(z-C) v^{(j+1)}(z), \quad j=0,1, \ldots, p-1$, understanding $L^{(j+2)} L^{(j+3)} \cdots L^{(p)}=I$ when $j=p-1$. From this and (20) (for $i=j+1$ ),

$$
L^{(j+2)} \cdots L^{(p)} U L^{(1)} \cdots L^{(j)} v^{(j)}(z)=(z-C) v^{(j+1)}(z), \quad j=0,1, \ldots, p-1,
$$

where $L^{(2)} \cdots L^{(p)} U v^{(0)}(z)=(z-C) v^{(1)}(z)$, this is, $L^{(1)} \cdots L^{(j)}=I$ when $j=0$.

On the other hand, it is easy to check that, for each $k \in \mathbb{N}$, the row $k$ of the infinite matrix $L^{(j+2)} \cdots L^{(p)} U L^{(1)} \cdots L^{(j)}$ is

$$
\left(g_{k, 1}^{(j)}, g_{k, 2}^{(j)}, \ldots, g_{k, k+1}^{(j)}, 0, \ldots\right) \text {, }
$$

being $g_{k, k+1}^{(j)}=1$ and the entries $g_{k, s}^{(j)}, s=1, \ldots, k$, independent on $z$. Moreover, $g_{k, s}^{(j)}=0$ for $s \leq k-p$ when $k>p$. In other words, $L^{(j+2)} \cdots L^{(p)} U L^{(1)} \cdots L^{(j)}$ is a $(p+1)$-banded Hessenberg matrix, where it is easy to see that $g_{k, k-p+1}^{(j)} \neq 0$ since $\gamma_{r} \neq 0(r \in \mathbb{N})$. Hence, taking into account (23),

$$
(z-C) P_{k-1}^{(j+1)}(z)=g_{k, k-p+1}^{(j)} P_{k-p}^{(j)}(z)+\cdots+g_{k, k+1}^{(j)} P_{k}^{(j)}(z)
$$

which drives to (18) when $k=n+1$. Note that, in this case,

$$
g_{n+1, n-p+2}^{(j)} \neq 0 \text {. }
$$

For $j=0$ and $i=p$, (20) becomes

$$
L v^{(p)}(z)=L^{(1)} \cdots L^{(p)} v^{(p)}(z)=v^{(0)}(z) .
$$

Therefore

$$
U v^{(0)}(z)=(z-C) v^{(p)}(z)
$$

(see (22) $)$ and, comparing the $(n+1)$-row in both sides of (25),

$$
(z-C) P_{n}^{(p)}(z)=\gamma_{n(p+1)+1} P_{n}(z)+P_{n+1}(z)
$$

The right hand side of (26) is a polynomial with a root in $z=C$. Thus

$$
\gamma_{n(p+1)+1}=-\frac{P_{n+1}(C)}{P_{n}(C)} .
$$

From (26) and (27) we arrive to (19). 
Remark 1 (18) and (19) coincide in the classic case $p=1$. Both relations extend [7, (7.3), pp. 35], this is,

$$
P_{n}^{(1)}(z)=\frac{P_{n+1}(z)-\frac{P_{n+1}(C)}{P_{n}(C)} P_{n}(z)}{z-C},
$$

where the sequence of Kernel polynomials $\left\{P_{n}^{(1)}\right\}$ are defined in terms of $\left\{P_{n}\right\}$. In this sense $\left\{P_{n}^{(j)}\right\}, j=1, \ldots, p$, are extensions of this classical sequence of Kernel polynomials.

The above remark justifies the following definition.

Definition 4 For each $j=1, \ldots, p$ the polynomials $\left\{P_{n}^{(j)}\right\}, n \in \mathbb{N}$, are called $j$-Kernel polynomials.

For each sequence $\left\{P_{n}^{(j)}\right\}, j=0,1, \ldots, p$, we denote by $\left\{\mathcal{L}_{n}^{(j)}\right\}$ the corresponding dual sequence (taking $\mathcal{L}_{n}^{(0)}=\mathcal{L}_{n}$ ). Equivalently to the behavior of the sequences of polynomials, the terms of the dual sequences are related to each other.

Lemma 3 With the above notation, for each $j=0,1 \ldots, p-1$ and $n=0,1, \ldots$ we have

$$
\begin{aligned}
\mathcal{L}_{n}^{(j+1)} & =\mathcal{L}_{n}^{(j)}+\gamma_{n(p+1)+j+2} \mathcal{L}_{n+1}^{(j)} \\
(z-C) \mathcal{L}_{n}^{(j)} & =\mathcal{L}_{n-1}^{(j+1)}+\sum_{s=0}^{p-1} g_{n+s+1, n+1}^{(j)} \mathcal{L}_{n+s}^{(j+1)} \\
(z-C) \mathcal{L}_{n} & =\mathcal{L}_{n-1}^{(p)}-\frac{P_{n+1}(C)}{P_{n}(C)} \mathcal{L}_{n}^{(p)}
\end{aligned}
$$

Proof.- In [6], the relation

$$
P_{m+1}^{(j)}=P_{m+1}^{(j+1)}+\gamma_{m(p+1)+j+2} P_{m}^{(j+1)}, \quad m=-1,0,1, \ldots,
$$

was proved (here, $\left.\gamma_{-(p+1)+j+2}=0\right)$. Then

$$
\begin{aligned}
& \mathcal{L}_{n}^{(j+1)}\left[P_{m+1}^{(j)}\right]=\mathcal{L}_{n}^{(j+1)}\left[P_{m+1}^{(j+1)}\right]+\gamma_{m(p+1)+j+2} \mathcal{L}_{n}^{(j+1)}\left[P_{m}^{(j+1)}\right]
\end{aligned}
$$

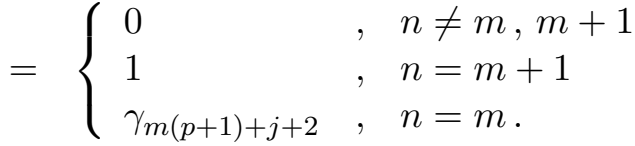

Moreover,

$$
\left(\mathcal{L}_{n}^{(j)}+\gamma_{n(p+1)+j+2} \mathcal{L}_{n+1}^{(j)}\right)\left[P_{m+1}^{(j)}\right]=\mathcal{L}_{n}^{(j)}\left[P_{m+1}^{(j)}\right]+\gamma_{n(p+1)+j+2} \mathcal{L}_{n+1}^{(j)}\left[P_{m+1}^{(j)}\right]
$$

also drives to (32). That is, both sides of (28) coincide on the basis $\left\{P_{m}^{(j)}\right\}, m \in \mathbb{N}$, of the space $\mathcal{P}$ of polynomials. Therefore (28) is verified.

For $m=0,1, \ldots$, taking into account (18),

$$
\begin{aligned}
& (z-C) \mathcal{L}_{n}^{(j)}\left[P_{m}^{(j+1)}\right]=\mathcal{L}_{n}^{(j)}\left[(z-C) P_{m}^{(j+1)}\right] \\
& =\mathcal{L}_{n}^{(j)}\left[P_{m+1}^{(j)}\right]+\sum_{s=0}^{p-1} g_{m+1, m-s+1}^{(j)} \mathcal{L}_{n}^{(j)}\left[P_{m-s}^{(j)}\right] \\
& = \begin{cases}0 & , \quad n \neq m+1, m, m-1, \ldots, m-p+1 . \\
1 & , \quad n=m+1 \\
g_{n+s+1, n+1}^{(j)} & , \quad n=m-s, \quad s=0,1, \ldots, p-1 .\end{cases}
\end{aligned}
$$


On the other hand,

$$
\left(\mathcal{L}_{n-1}^{(j+1)}+\sum_{s=0}^{p-1} g_{n+s+1, n+1}^{(j)} \mathcal{L}_{n+s}^{(j+1)}\right)\left[P_{m}^{(j+1)}\right]=\mathcal{L}_{n-1}^{(j+1)}\left[P_{m}^{(j+1)}\right]+\sum_{s=0}^{p-1} g_{n+s+1, n+1}^{(j)} \mathcal{L}_{n+s}^{(j+1)}\left[P_{m}^{(j+1)}\right]
$$

coincides with (33) for each $m \in \mathbb{N}$. Therefore, (29) holds. We underline that this is true even if $n=0$ in (29), understanding $\mathcal{L}_{-1}^{(j+1)}=0$ in this case.

As in (28)-(29), we apply both sides of (30) to a basis of polynomials. Then using (19),

$$
\begin{aligned}
& (z-C) \mathcal{L}_{n}\left[P_{m}^{(p)}\right]=\mathcal{L}_{n}\left[(z-C) P_{m}^{(p)}\right] \\
& =\mathcal{L}_{n}\left[P_{m+1}-\frac{P_{m+1}(C)}{P_{m}(C)} P_{m}\right] \\
& =\mathcal{L}_{n}\left[P_{m+1}\right]-\frac{P_{m+1}(C)}{P_{m}(C)} \mathcal{L}_{n}\left[P_{m}\right]=\left\{\begin{array}{cl}
0 & , \quad n \neq m, m+1 \\
1 & , \quad n=m+1 \\
-\frac{P_{m+1}(C)}{P_{m}(C)} \quad, & n=m .
\end{array}\right.
\end{aligned}
$$

Further,

$$
\left(\mathcal{L}_{n-1}^{(p)}-\frac{P_{n+1}(C)}{P_{n}(C)} \mathcal{L}_{n}^{(p)}\right)\left[P_{m}^{(p)}\right]=\mathcal{L}_{n-1}^{(p)}\left[P_{m}^{(p)}\right]-\frac{P_{n+1}(C)}{P_{n}(C)} \mathcal{L}_{n}^{(p)}\left[P_{m}^{(p)}\right]
$$

which produces exactly the same result as in (34). Then (30) is proved.

In the classic case $p=1$, the functionals of orthogonality $\nu$ and $\nu^{(1)}$, associated respectively with $\left\{P_{n}\right\}$ and the Kernel polynomials $\left\{P_{n}^{(1)}\right\}$, are related by

$$
\nu^{(1)}=(z-C) \nu .
$$

The next result extends this fact to the general case $p \in \mathbb{N}$. In fact, this lemma is equivalently to Theorem 1 in the case $j=p$.

Lemma 4 Let $\nu=\left(\nu_{1}, \nu_{2}, \ldots, \nu_{p}\right)$ be a vector of $p$-orthogonality for $\left\{P_{n}\right\}$. Then

$$
\nu^{(p)}=\left((z-C) \nu_{1},(z-C) \nu_{2}, \ldots,(z-C) \nu_{p}\right)
$$

is a vector of p-orthogonality for the p-Kernel polynomials $\left\{P_{n}^{(p)}\right\}$.

Proof.- Due to (19), for each $r=1,2, \ldots, p$ we have

$$
\begin{aligned}
\left((z-C) \nu_{r}\right)\left[z^{k} P_{m p+i}^{(p)}\right] & =\nu_{r}\left[z^{k}(z-C) P_{m p+i}^{(p)}\right]=\nu_{r}\left[z^{k}\left(P_{m p+i+1}-\frac{P_{m p+i+1}(C)}{P_{m p+i}(C)} P_{m p+i}\right)\right] \\
& =\nu_{r}\left[z^{k} P_{m p+i+1}\right]-\frac{P_{m p+i+1}(C)}{P_{m p+i}(C)} \nu_{r}\left[z^{k} P_{m p+i}\right]
\end{aligned}
$$

where

$$
\left\{\begin{aligned}
\nu_{r}\left[z^{k} P_{m p+i+1}\right] & =0 \quad, \quad k \geq 0, & k p+r \leq m p+i+1 \\
\nu_{r}\left[z^{k} P_{m p+i}\right] & =0 \quad, \quad k \geq 0, & k p+r \leq m p+i
\end{aligned}\right.
$$


Therefore, if $k p+r \leq m p+i$ then (35) holds and

$$
\left((z-C) \nu_{r}\right)\left[z^{k} P_{m p+i}^{(p)}\right]=0, \quad k \geq 0, \quad k p+r \leq m p+i .
$$

Moreover, using (18),

$$
\left((z-C) \nu_{r}\right)\left[z^{k} P_{k p+r-1}^{(p)}\right]=\nu_{r}\left[z^{k} P_{k p+r}\right]-\frac{P_{k p+r}(C)}{P_{k p+r-1}(C)} \nu_{r}\left[z^{k} P_{k p+r-1}\right]
$$

where $\nu_{r}\left[z^{k} P_{k p+r}\right]=0$ (see (35)) and $\nu_{r}\left[z^{k} P_{k p+r-1}\right] \neq 0$ (see (2) ). This is,

$$
\left((z-C) \nu_{r}\right)\left[z^{k} P_{k p+r-1}^{(p)}\right]=-\frac{P_{k p+r}(C)}{P_{k p+r-1}(C)} \nu_{r}\left[z^{k} P_{k p+r-1}\right] \neq 0 .
$$

This proves that $(z-C) \nu_{r}$ is the $r$-th entry of a vector of $p$-orthogonality associated with the sequence of polynomials $\left\{P_{n}^{(p)}\right\}$ and, consequently, $\nu^{(p)}$ is one of such vectors.

Remark 2 We underline that, in the case $j=p$, we have proved that the statement of Theorem 1 is verified independently on the condition (16).

Lemma 5 For each $j=0,1, \ldots, p$, let $\nu^{(j)}=\left(\nu_{1}^{(j)}, \nu_{2}^{(j)} \ldots, \nu_{p}^{(j)}\right)$ be a vector of $p$ orthogonality for $\left\{P_{n}^{(j)}\right\}$. Then, for $j=0,1, \ldots, p-1$ we have:

(a) $\tilde{\nu}^{(j+1)}=\left(\nu_{1}^{(j+1)}, \ldots, \nu_{p-1}^{(j+1)},(z-C) \nu_{1}^{(j)}\right)$ is a vector of $p$-orthogonality for $\left\{P_{n}^{(j+1)}\right\}$.

(b) $\tilde{\nu}^{(j)}=\left(\nu_{1}^{(j)}, \nu_{1}^{(j+1)} \ldots, \nu_{p-1}^{(j+1)}\right)$ is a vector of $p$-orthogonality for $\left\{P_{n}^{(j)}\right\}$.

Proof.- In the first place, because the first entries of the vector $\tilde{\nu}^{(j+1)}$ coincide with the corresponding to $\nu^{(j+1)}$, to prove $(a)$ it is enough to check

$$
\begin{aligned}
(z-C) \nu_{1}^{(j)}\left[z^{k} P_{n}^{(j+1)}\right] & =0, \quad k p+p \leq n, \\
(z-C) \nu_{1}^{(j)}\left[z^{k} P_{(k+1) p-1}^{(j+1)}\right] & \neq 0 .
\end{aligned}
$$

Indeed, using (18) of Theorem 2 ,

$$
(z-C) \nu_{1}^{(j)}\left[z^{k} P_{n}^{(j+1)}\right]=\nu_{1}^{(j)}\left[z^{k} P_{n+1}^{(j)}\right]+\sum_{s=0}^{p-1} g_{n+1, n-s+1}^{(j)} \nu_{1}^{(j)}\left[z^{k} P_{n-s}^{(j)}\right]
$$

where $\nu_{1}^{(j)}\left[z^{k} P_{n+1}^{(j)}\right]=0$ for $k p+1 \leq n+1$ and $\nu_{1}^{(j)}\left[z^{k} P_{n-s}^{(j)}\right]=0$ for $k p+1 \leq n-s, s=$ $0,1, \ldots, p-1$.

Then,

$$
(z-C) \nu_{1}^{(j)}\left[x^{k} P_{n}^{(j+1)}\right]=0
$$

for $k p+1 \leq n-p+1$ or, what is the same, (36) holds. 
For a similar reason,

$$
\begin{aligned}
(z-C) \nu_{1}^{(j)}\left[z^{k} P_{k p+p-1}^{(j+1)}\right] & =\nu_{1}^{(j)}\left[z^{k} P_{k p+p}^{(j)}\right]+\sum_{s=0}^{p-1} g_{k p+p, k p-s+p}^{(j)} \nu_{1}^{(j)}\left[z^{k} P_{k p-s+p-1}^{(j)}\right] \\
& =g_{k p+p, k p+1}^{(j)} \nu_{1}^{(j)}\left[z^{k} P_{k p}^{(j)}\right] \neq 0
\end{aligned}
$$

which, taking into account (24), gives (37). Thus $(a)$ is verified.

In the second place, take $r \in\{1, \ldots, p-1\}, k \in\{0,1, \ldots$,$\} and n \in \mathbb{N}$. Using (31),

$$
\nu_{r}^{(j+1)}\left[z^{k} P_{n}^{(j)}\right]=\nu_{r}^{(j+1)}\left[z^{k} P_{n}^{(j+1)}\right]+\gamma_{(n-1)(p+1)+j+2} \nu_{r}^{(j+1)}\left[z^{k} P_{n-1}^{(j+1)}\right] .
$$

In (2) we see

$$
\nu_{r}^{(j+1)}\left[z^{k} P_{n}^{(j+1)}\right]=\nu_{r}^{(j+1)}\left[z^{k} P_{n-1}^{(j+1)}\right]=0, \quad k p+r \leq n-1 .
$$

Hence (38) implies

$$
\nu_{r}^{(j+1)}\left[z^{k} P_{n}^{(j)}\right]=0, \quad k p+r+1 \leq n .
$$

From (38)-(39), taking $n=k p+r$,

$$
\nu_{r}^{(j+1)}\left[z^{k} P_{k p+r}^{(j)}\right]=\gamma_{(k p+r-1)(p+1)+j+2} \nu_{r}^{(j+1)}\left[z^{k} P_{k p+r-1}^{(j+1)}\right] \neq 0 .
$$

Since (2), we have that (40) and (41) give $\nu_{r}^{(j+1)}=\widetilde{\nu}_{r+1}^{(j)}$, which is the $(r+1)$-th entry of vector of $p$-orthogonality for $\left\{P_{n}^{(j)}\right\}, n \in \mathbb{N}$ (we recall that $r+1 \leq p$ ).

\section{Proof of Theorem 1}

Through Lemma 4 and Remark 2, the result is verified for $j=p$, independent on the factorization (11). Then we want to find a Darboux factorization (11) such that (17) is a vector of $p$-orthogonality for the corresponding sequence $\left\{P_{n}^{(j)}\right\}$ of polynomials when $j=1, \ldots, p-1$.

We proceed recursively on $j=1,2, \ldots, p$.

\subsection{First step: $j=1$}

In this case, in (17) we have the vector of functionals

$$
\nu^{(1)}=\left(\nu_{2}, \ldots, \nu_{p},(z-C) \nu_{1}\right) .
$$

Due to Lemma 5, to prove Theorem 1 it is sufficient to show that $\nu_{2}, \ldots, \nu_{p}$ are the first $p-1$ entries of a vector of $p$-orthogonality for $\left\{P_{n}^{(j)}\right\}$, where this sequence of polynomials is corresponding to some Darboux transformation $J^{(1)}$ of $J-C I$. In this step, we choose $L^{(1)}$ appropriately for our goal. This is, we will see how to fix the entries

$$
\gamma_{2}, \gamma_{p+3}, \ldots, \gamma_{(p-1) p}
$$


of $L^{(1)}$ in (10) with the purpose to define $\mathcal{L}_{m}^{(1)}$ as in (28) and to find $\lambda_{m, k}^{(1)} \in \mathbb{C}, k=$ $0,1, \ldots, m-1$, and $\lambda_{m, m-1}^{(1)} \neq 0$ such that

$$
\nu_{m+1}=\sum_{k=0}^{m-1} \lambda_{m, k}^{(1)} \mathcal{L}_{k}^{(1)}, \quad m=1,2, \ldots, p-1
$$

$($ see (44)).

Because $\nu$ is a vector of $p$-orthogonality for $\left\{P_{n}\right\}$, we know

$$
\nu_{m+1}=\sum_{k=0}^{m} \lambda_{m+1, k} \mathcal{L}_{k}, \quad m=1,2, \ldots, p-1 .
$$

For any Darboux factorization, (28) holds taking $j=0$. From this (43) is equivalent to

$$
\nu_{m+1}=\lambda_{m, 0}^{(1)} \mathcal{L}_{0}+\sum_{k=1}^{m-1}\left(\gamma_{(k-1)(p+1)+2} \lambda_{m, k-1}^{(1)}+\lambda_{m, k}^{(1)}\right) \mathcal{L}_{k}+\gamma_{(m-1)(p+1)+2} \lambda_{m, m-1}^{(1)} \mathcal{L}_{m} .
$$

Comparing the last expression with (44) for $m=1, \ldots, p-1$, we have

$$
\begin{aligned}
\lambda_{m, 0}^{(1)} & =\lambda_{m+1,0}, \\
\gamma_{(k-1)(p+1)+2} \lambda_{m, k-1}^{(1)}+\lambda_{m, k}^{(1)} & =\lambda_{m+1, k}, \quad k=1, \ldots, m-1, \\
\gamma_{(m-1)(p+1)+2} \lambda_{m, m-1}^{(1)} & =\lambda_{m+1, m} .
\end{aligned}
$$

We rewrite (45)-(47) as

$$
L_{m+1}^{(1)}\left(\begin{array}{c}
\lambda_{m, 0}^{(1)} \\
\lambda_{m, 1}^{(1)} \\
\vdots \\
\lambda_{m, m-1}^{(1)} \\
0
\end{array}\right)=\left(\begin{array}{c}
\lambda_{m+1,0} \\
\lambda_{m+1,1} \\
\vdots \\
\lambda_{m+1, m-1} \\
\lambda_{m+1, m}
\end{array}\right), m=1, \ldots, p-1 .
$$

In other words, (45)-(46) is

$$
\left(\begin{array}{c}
\lambda_{m, 0}^{(1)} \\
\lambda_{m, 1}^{(1)} \\
\vdots \\
\lambda_{m, m-1}^{(1)}
\end{array}\right)=\left(L_{m}^{(1)}\right)^{-1}\left(\begin{array}{c}
\lambda_{m+1,0} \\
\lambda_{m+1,1} \\
\vdots \\
\lambda_{m+1, m-1}
\end{array}\right), m=1, \ldots, p-1
$$

or, what is the same,

$$
\begin{aligned}
& \lambda_{m, k-1}^{(1)}=\lambda_{m+1, k-1}-\gamma_{(k-2)(p+1)+2} \lambda_{m+1, k-2}+\gamma_{(k-3)(p+1)+2} \gamma_{(k-2)(p+1)+2} \lambda_{m+1, k-3}- \\
& \cdots+(-1)^{k-1} \gamma_{2} \gamma_{(p+1)+2} \cdots \gamma_{(k-2)(p+1)+2} \lambda_{m+1,0}, \quad 1 \leq k \leq m, \quad 1 \leq m \leq p-1 .
\end{aligned}
$$


Furthermore, taking into account the expression of the last row of $\left(L_{m+1}^{(1)}\right)^{-1}$, the following relation joint with (49) are equivalent to (48),

$$
\begin{array}{r}
\lambda_{m+1, m}-\gamma_{(m-1)(p+1)+2} \lambda_{m+1, m-1}+\cdots+(-1)^{m} \gamma_{2} \gamma_{(p+1)+2} \cdots \gamma_{(m-1)(p+1)+2} \lambda_{m+1,0}= \\
m=1, \ldots, p-1 .
\end{array}
$$

Therefore, the proof of the case $j=1$ is reduced to find entries $\gamma_{2}, \ldots, \gamma_{(p-1) p}$ of $L^{(1)}$ verifying the condition (50) such that the coefficients $\lambda_{m, k-1}^{(1)}, k=1, \ldots, m$, provided in (49) define a vector of functionals

$$
\nu^{(1)}=\left(\nu_{1}^{(1)}, \ldots, \nu_{p-1}^{(1)},(z-C) \nu_{1}\right)
$$

as in (42). We proceed recursively for $m=1,2, \ldots, p-1$.

For $m=1$ in (50) we have $\lambda_{2,1}-\gamma_{2} \lambda_{2,0}=0$, where we know from (16) that $\Delta_{1}=$ $\lambda_{2,0} \neq 0$. Then, taking $\Delta_{0}:=1$, we can define

$$
\gamma_{2}=\lambda_{2,1} \frac{\Delta_{0}}{\Delta_{1}}
$$

Moreover, since (49) we define $\lambda_{1,0}^{(1)}=\lambda_{2,0}$ and $\nu_{1}^{(1)}=\lambda_{2,0} \mathcal{L}_{0}$ has been constructed.

Now we will to prove that the first entries of $L^{(1)}$ can be choosen as

$$
\gamma_{(m-1)(p+1)+2}=\lambda_{m+1, m} \frac{\Delta_{m-1}}{\Delta_{m}}, \quad m=1,2, \ldots, p-1 .
$$

Indeed, (51) is verified for $m=1$. Assume that (51) holds for $m \leq s<p-1$. Assume also that

$$
\gamma_{2}, \gamma_{(p+1)+2}, \ldots, \gamma_{(s-1)(p+1)+2}
$$

have been choosen verifying (50). Then $\gamma_{s(p+1)+2}$ can be defined taking $m=s+1$ in (50), this is,

$$
\begin{aligned}
0 & =\lambda_{s+2, s+1}-\gamma_{s(p+1)+2}\left[\lambda_{s+2, s}-\gamma_{(s-1)(p+1)+2} \lambda_{s+2, s-1}+\right. \\
& \left.+\cdots+(-1)^{s+1} \gamma_{2} \gamma_{(p+1)+2} \cdots \gamma_{(s-1)(p+1)+2} \lambda_{s+2,0}\right]
\end{aligned}
$$

where, from (51), we see that

$$
\Delta_{s}\left[\lambda_{s+2, s}-\gamma_{(s-1)(p+1)+2} \lambda_{s+2, s-1}+\cdots+(-1)^{s+1} \gamma_{2} \gamma_{(p+1)+2} \ldots \gamma_{(s-1)(p+1)+2} \lambda_{s+2,0}\right]
$$

is the development of the determinant $\Delta_{s+1}$ by its last column. Thus

$$
\gamma_{s(p+1)+2}=\lambda_{s+2, s+1} \frac{\Delta_{s}}{\Delta_{s+1}}
$$

and (51) is verified in $m=s+1$ and, consequently, for all $m=1,2, \ldots, p-1$.

In this way the entries

$$
\gamma_{2}, \gamma_{(p+1)+2}, \ldots, \gamma_{(p-1) p}
$$

of $L^{(1)}$ are chosen verifying (51) and the coefficients $\lambda_{m, k-1}^{(1)}, k=1, \ldots, m$, given in (49) define the vector of orthogonality

$$
\nu^{(1)}=\left(\nu_{1}^{(1)}, \ldots, \nu_{p-1}^{(1)},(z-C) \nu_{1}\right)
$$

for a new sequence of polynomials $\left\{P_{n}^{(1)}\right\}$. 


\subsection{Steps $2,3, \ldots, p-1$.}

In each one of the following steps, we want to find the first appropriate entries of the corresponding bidiagonal matrix. This is, in the step $j+1$, for $j=1,2, \ldots, p-1$, we assume that for each $s=1,2, \ldots, j$ the entries

$$
\gamma_{s+1}, \gamma_{(p+1)+s+1}, \ldots, \gamma_{(p-s-1)(p+1)+s+1}
$$

of $L^{(s)}$ have been defined such that

$$
\nu^{(s)}=\left(\nu_{s+1}, \ldots, \nu_{p},(z-C) \nu_{1}, \ldots,(z-C) \nu_{s}\right)
$$

is a vector of $p$-orthogonality for $\left\{P_{n}^{(s)}\right\}$. Then, we want to find the first $p-j-1$ entries

$$
\gamma_{j+2}, \gamma_{(p+1)+j+2}, \ldots, \gamma_{(p-j-1) p}
$$

of $L^{(j+1)}$ such that $\nu^{(j+1)}$ in (17) is a vector of $p$-orthogonality for the corresponding sequence $\left\{P_{n}^{(j+1)}\right\}$. Due to the case $j+1=p$ is solved in Lemma 4, we assume $j \in$ $\{1,2, \ldots, p-2\}$ in the following. We differentiate two kind of entries in $\nu^{(j+1)}$. This is, we denote

$$
\nu^{(j+1)}=\left(\nu_{1}^{(j+1)}, \ldots, \nu_{p}^{(j+1)}\right)
$$

where

$$
\nu_{k}^{(j+1)}= \begin{cases}\nu_{j+k+1} & , k=1, \ldots, p-j-1 \\ (z-C) \nu_{j+k+1-p} & , k=p-j, \ldots, p .\end{cases}
$$

In the first place, we analyze the entries $\nu_{k}^{(j+1)}, k=1, \ldots, p-j-1$, for which we want to define $\mathcal{L}_{s}^{(j+1)}, s=0, \ldots, k-1$, as in (28) and to find $\lambda_{k, 0}^{(j+1)}, \ldots, \lambda_{k, k-1}^{(j+1)}$ such that

$$
\nu_{k}^{(j+1)}=\sum_{s=0}^{k-1} \lambda_{k, s}^{(j+1)} \mathcal{L}_{s}^{(j+1)} .
$$

Since (17) and (52) we know that

$$
\nu_{k}^{(j+1)}=\nu_{k+1}^{(j)}, \quad k=1, \ldots, p-j-1 .
$$

Hence,

$$
\nu_{k}^{(j+1)}=\sum_{r=0}^{k} \lambda_{k+1, r}^{(j)} \mathcal{L}_{r}^{(j)}, \quad k=1, \ldots, p-j-1 .
$$

Using (28) in (53),

$$
\begin{aligned}
\nu_{k}^{(j+1)} & =\sum_{s=0}^{k-1} \lambda_{k, s}^{(j+1)}\left(\mathcal{L}_{s}^{(j)}+\gamma_{s(p+1)+j+2} \mathcal{L}_{s+1}^{(j)}\right)= \\
& =\sum_{s=0}^{k-1} \lambda_{k, s}^{(j+1)} \mathcal{L}_{s}^{(j)}+\sum_{s=1}^{k} \gamma_{(s-1)(p+1)+j+2} \lambda_{k, s-1}^{(j+1)} \mathcal{L}_{s}^{(j)}= \\
=\lambda_{k, 0}^{(j+1)} \mathcal{L}_{0}^{(j)} & +\sum_{s=1}^{k-1}\left(\lambda_{k, s}^{(j+1)}+\gamma_{(s-1)(p+1)+j+2} \lambda_{k, s-1}^{(j+1)}\right) \mathcal{L}_{s}^{(j)}+\gamma_{(k-1)(p+1)+j+2} \lambda_{k, k-1}^{(j+1)} \mathcal{L}_{k}^{(j)}
\end{aligned}
$$


Comparing with (54),

$$
\lambda_{k+1, s}^{(j)}= \begin{cases}\lambda_{k, 0}^{(j+1)}, & s=0, \\ \lambda_{k, s}^{(j+1)}+\gamma_{(s-1)(p+1)+j+2} \lambda_{k, s-1}^{(j+1)}, & s=1, \ldots, k-1, \\ \gamma_{(k-1)(p+1)+j+2} \lambda_{k, k-1}^{(j+1)}, & s=k .\end{cases}
$$

Similarly to what was done in (45)-(47), (55) can be rewritten as

$$
L_{k+1}^{(j+1)}\left(\begin{array}{c}
\lambda_{k, 0}^{(j+1)} \\
\lambda_{k, 1}^{(j+1)} \\
\vdots \\
\lambda_{k, k-1}^{(j+1)} \\
0
\end{array}\right)=\left(\begin{array}{c}
\lambda_{k+1,0}^{(j)} \\
\lambda_{k+1,1}^{(j)} \\
\vdots \\
\lambda_{k+1, k-1}^{(j)} \\
\lambda_{k+1, k}^{(j)}
\end{array}\right), k=1, \ldots, p-j-1
$$

or, what is the same,

$$
L_{k}^{(j+1)}\left(\begin{array}{c}
\lambda_{k, 0}^{(j+1)} \\
\lambda_{k, 1}^{(j+1)} \\
\vdots \\
\lambda_{k, k-1}^{(j+1)}
\end{array}\right)=\left(\begin{array}{c}
\lambda_{k+1,0}^{(j)} \\
\lambda_{k+1,1}^{(j)} \\
\vdots \\
\lambda_{k+1, k-1}^{(j)}
\end{array}\right), k=1, \ldots, p-j-1
$$

with the additional condition

$$
\lambda_{k+1, k}^{(j)}=\gamma_{(k-1)(p+1)+j+2} \lambda_{k, k-1}^{(j+1)}, \quad k=1, \ldots, p-j-1 .
$$

Therefore, finding

$$
\lambda_{k, 0}^{(j+1)}, \ldots, \lambda_{k, k-1}^{(j+1)}
$$

as in (53) comes down to choose the entries

$$
\gamma_{j+2}, \ldots, \gamma_{(p-j-1) p}
$$

of $L_{p-j}^{(j+1)}$ with the aim of (157)-(58) take place. We note that (57) defines the coefficients $\lambda_{k, s}^{(j+1)}, s=0, \ldots, k-1$, because $L_{p-j}^{(j+1)}$ is an invertible matrix. Then, (58) is equivalent to the fact that the last row of $\left(L_{k+1}^{(j+1)}\right)^{-1}$ multiplied by $\left(\lambda_{k+1,0}^{(j)}, \ldots, \lambda_{k+1, k}^{(j)}\right)$ vanishes. This is,

$$
\begin{aligned}
\lambda_{k+1, k}^{(j)} & -\lambda_{k+1, k-1}^{(j)} \gamma_{(k-1)(p+1)+j+2}+\lambda_{k+1, k-2}^{(j)} \gamma_{(k-2)(p+1)+j+2} \gamma_{(k-1)(p+1)+j+2} \\
-\cdots & +(-1)^{k} \lambda_{k+1,0}^{(j)} \gamma_{j+2} \gamma_{(p+1)+j+2} \cdots \gamma_{(k-1)(p+1)+j+2}=0, \quad k=1,2, \ldots, p-j .
\end{aligned}
$$

Now we show that in the above conditions the following matrix equality is verified for 
$j=1,2, \ldots, p-1$ and $s=1,2, \ldots$,

$$
\begin{gathered}
L_{s}^{(1)} \ldots L_{s}^{(j)}\left(\begin{array}{cccccc}
\lambda_{2,0}^{(j)} & \lambda_{3,0}^{(j)} & \cdots & \cdots & \cdots & \lambda_{s+1,0}^{(j)} \\
\lambda_{2,1}^{(j)} & \lambda_{3,1}^{(j)} & \cdots & \cdots & \cdots & \lambda_{s+1,1}^{(j)} \\
0 & \lambda_{3,2}^{(j)} & \ddots & & & \vdots \\
\vdots & 0 & \ddots & \ddots & & \\
\vdots & \vdots & \ddots & & & \vdots \\
0 & 0 & \cdots & 0 & \lambda_{s, s-1}^{(j)} & \lambda_{s+1, s-1}^{(j)}
\end{array}\right) \\
=\left(\begin{array}{ccccccc}
\lambda_{j+2,0} & \lambda_{j+3,0} & \cdots & \cdots & \lambda_{s, 0} & \cdots & \lambda_{j+s+1,0} \\
\vdots & \vdots & & & \vdots & & \\
\lambda_{j+2, j+1} & \lambda_{j+3, j+1} & \cdots & \cdots & \lambda_{s, j+1} & \cdots & \lambda_{j+s+1, j+1} \\
0 & \lambda_{j+3, j+2} & \ddots & & \vdots & & \vdots \\
\vdots & 0 & \ddots & \ddots & & \cdots & \\
\vdots & \vdots & \ddots & \ddots & \vdots & & \vdots \\
0 & 0 & \cdots & 0 & \lambda_{s, s-1} & \cdots & \lambda_{j+s+1, s-1}
\end{array}\right) .
\end{gathered}
$$

In fact, as in (56), it is easy to see

$$
L_{k+1}^{(r)}\left(\begin{array}{c}
\lambda_{k, 0}^{(r)} \\
\lambda_{k, 1}^{(r)} \\
\vdots \\
\lambda_{k, k-1}^{(r)} \\
0
\end{array}\right)=\left(\begin{array}{c}
\lambda_{k+1,0}^{(r-1)} \\
\lambda_{k+1,1}^{(r-1)} \\
\vdots \\
\lambda_{k+1, k-1}^{(r-1)} \\
\lambda_{k+1, k}^{(r-1)}
\end{array}\right), k=1, \ldots, p-r, \quad r=1,2, \ldots, j+1 .
$$

Then, considering each infinite matrix $L^{(r)}$,

$$
L^{(r)}\left(\begin{array}{c}
\lambda_{k, 0}^{(r)} \\
\lambda_{k, 1}^{(r)} \\
\vdots \\
\lambda_{k, k-1}^{(r)} \\
0 \\
0 \\
\vdots
\end{array}\right)=\left(\begin{array}{c}
\lambda_{k+1,0}^{(r-1)} \\
\lambda_{k+1,1}^{(r-1)} \\
\vdots \\
\lambda_{k+1, k-1}^{(r-1)} \\
\lambda_{k+1, k}^{(r-1)} \\
0 \\
\vdots
\end{array}\right), k=1, \ldots, p-r, \quad r=1,2, \ldots, j+1
$$

Applying iteratively $L^{(r)}, \ldots, L^{(1)}$ to (61) and then taking $r=j$ we arrive to

$$
L^{(1)} \cdots L^{(j)}\left(\begin{array}{c}
\lambda_{k, 0}^{(j)} \\
\vdots \\
\lambda_{k, k-1}^{(r j)} \\
0 \\
\vdots
\end{array}\right)=\left(\begin{array}{c}
\lambda_{j+k, 0} \\
\vdots \\
\vdots \\
\lambda_{j+k, j+k-1} \\
0 \\
\vdots
\end{array}\right), k=1, \ldots, p-j .
$$


From this, for $s \in \mathbb{N}$, taking $k=2,3, \ldots, s+1$,

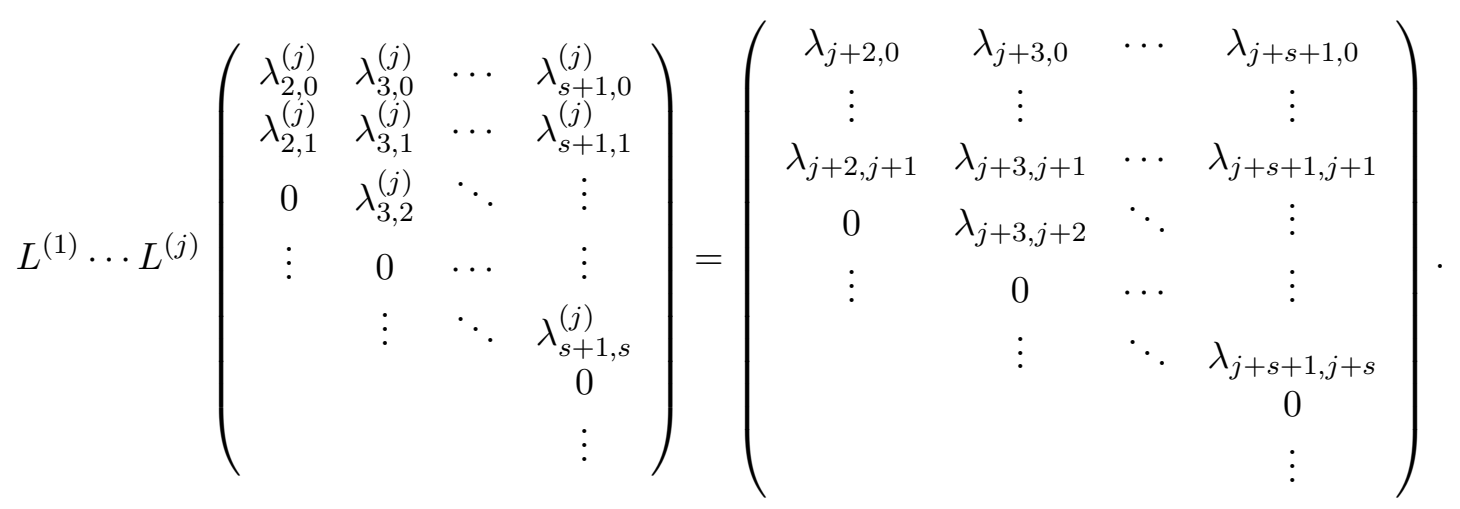

Therefore, due to $L^{(1)} \cdots L^{(j)}$ is an infinite lower triangular matrix, we have

$$
\left(L^{(1)} \cdots L^{(j)}\right)_{s}=L_{s}^{(1)} \cdots L_{s}^{(j)}
$$

and we arrive to (60).

As a consequence of (60),

$$
\Delta_{s}^{(j)}=\left|\begin{array}{cccccc}
\lambda_{2,0}^{(j)} & \lambda_{3,0}^{(j)} & \cdots & \cdots & \ldots & \lambda_{s+1,0}^{(j)} \\
\lambda_{2,1}^{(j)} & \lambda_{3,1}^{(j)} & \cdots & \cdots & \ldots & \lambda_{s+1,1}^{(j)} \\
0 & \lambda_{3,2}^{(j)} & \ddots & & & \vdots \\
\vdots & 0 & \ddots & \ddots & & \\
\vdots & \vdots & \ddots & & & \vdots \\
0 & 0 & \cdots & 0 & \lambda_{s, s-1}^{(j)} & \lambda_{s+1, s-1}^{(j)}
\end{array}\right|, \quad j=1, \ldots, p-1, \quad s \in \mathbb{N} .
$$

Taking $k=1$ in (59), we have $\lambda_{2,1}^{(j)}-\lambda_{2,0}^{(j)} \gamma_{j+2}=0$. Because we know that $\lambda_{2,1}^{(j)} \neq 0$ (see (44)) and $\Delta_{1}^{(j)}=\lambda_{2,0}^{(j)} \neq 0$, it is possible to take

$$
\gamma_{j+2}=\lambda_{2,1}^{(j)} \frac{\Delta_{0}^{(j)}}{\Delta_{1}^{(j)}}
$$

(where we define $\Delta_{0}^{(j)}:=1$ ). Iterating the procedure, assuming

$$
\gamma_{m(p+1)+j+2}=\lambda_{m+2, m+1}^{(j)} \frac{\Delta_{m}^{(j)}}{\Delta_{m+1}^{(j)}}, \quad m=0,1, \ldots, s,
$$

with $s<p-j-2$ and taking $k=s+2$ in (59),

$$
\begin{gathered}
\lambda_{s+3, s+2}^{(j)}-\gamma_{(s+1)(p+1)+j+2}\left[\lambda_{s+3, s+1}^{(j)}-\lambda_{s+3, s}^{(j)} \gamma_{s(p+1)+j+2}+\right. \\
\left.\cdots+(-1)^{s+1} \lambda_{s+3,0}^{(j)} \gamma_{j+2} \ldots \gamma_{s(p+1)+j+2}\right]=0 .
\end{gathered}
$$


where $\gamma_{(s+1)(p+1)+j+2}$ can be defined as

$$
\gamma_{(s+1)(p+1)+j+2}=\lambda_{s+3, s+2}^{(j)} \frac{\Delta_{s+1}^{(j)}}{\Delta_{s+1}^{(j)}\left[\lambda_{s+3, s+1}^{(j)}-\cdots+(-1)^{s+1} \lambda_{s+3,0}^{(j)} \gamma_{j+2} \cdots \gamma_{s(p+1)+j+2}\right]} .
$$

Further, from (62) it is easy to check that the denominator in (63) is the development of the determinant $\Delta_{s+2}^{(j)}$ by its last column. Thus $\gamma_{m(p+1)+j+2}$ can be defined as in (62) for $m=0,1, \ldots, p-j-2$.

Finally, we study the entries $\nu_{k}^{(j+1)}, k=p-j, \ldots, p$ of $\nu^{(j+1)}$. We want to prove

$$
\begin{aligned}
& (z-C) \nu_{j+k+1-p}\left[z^{s} P_{n}^{(j+1)}\right]=0, \quad n \geq s p+k \\
& (z-C) \nu_{j+k+1-p}\left[z^{s} P_{s p+k-1}^{(j+1)}\right] \neq 0,
\end{aligned}
$$

because this means, from (2), that $(z-C) \nu_{j+k+1-p}$ is the entry $k$ of a vector of $p$ orthogonality for $\left\{P_{n}^{(j+1)}\right\}$.

Lemma 4 implies

$$
\begin{aligned}
(z-C) \nu_{j+k+1-p}\left[z^{s} P_{m}^{(p)}\right] & =0, \quad m \geq s p+j+k+1-p \\
(z-C) \nu_{j+k+1-p}\left[z^{s} P_{s p+j+k-p}^{(p)}\right] & \neq 0 .
\end{aligned}
$$

In addition, since (31), for any Darboux factorization,

$$
\left(\begin{array}{c}
P_{0}^{(j+1)} \\
P_{1}^{(j+1)} \\
\vdots
\end{array}\right)=L^{(j+2)}\left(\begin{array}{c}
P_{0}^{(j+2)} \\
P_{1}^{(j+2)} \\
\vdots
\end{array}\right)=\cdots=L^{(j+2)} L^{(j+3)} \ldots L^{(p)}\left(\begin{array}{c}
P_{0}^{(p)} \\
P_{1}^{(p)} \\
\vdots
\end{array}\right) .
$$

Thus, $P_{n}^{(j+1)}$ can be expressed in terms of the entries in the $(n+1)$-th row of $L^{(j+2)} L^{(j+3)} \ldots L^{(p)}$ and the sequence $\left\{P_{n}^{(p)}\right\}$. From this, taking into account that $L^{(j+2)} L^{(j+3)} \ldots L^{(p)}$ is a lower triangular $(p-j)$-banded matrix, we see

$$
P_{n}^{(j+1)}=\sum_{r=n+j-p+1}^{n} \alpha_{r} P_{r}^{(p)}, \quad n \geq 0 \quad\left(\text { with } \alpha_{n}=1\right) .
$$

Hence,

$$
(z-C) \nu_{j+k+1-p}\left[z^{s} P_{n}^{(j+1)}\right]=\sum_{r=n+j-p+1}^{n} \alpha_{r}(z-C) \nu_{j+k+1-p}\left[z^{s} P_{r}^{(p)}\right]
$$

and, using (66), we see that each term on the right hand side of (67) vanishes when $n \geq s p+k$. Thus (64) is verified.

To see (65), if $n=s p+k-1$ in (67), then using (66) on the right hand side of (67) we have

$$
\sum_{r=s p+k+j-p}^{s p+k-1} \alpha_{r}(z-C) \nu_{j+k+1-p}\left[z^{s} P_{r}^{(p)}\right]=\alpha_{s p+k+j-p}(z-C) \nu_{j+k+1-p}\left[z^{s} P_{s p+j+k-p}^{(p)}\right] \neq 0 .
$$


Remark 3 Note that, if $\Delta_{s}^{(j)} \neq 0$ for $s=0,1, \ldots, p-m-1$ and $j=1, \cdots, m-1$, then there exist $m$ bidiagonal matrices $L^{(1)}, \ldots, L^{(m)}$ such that

$$
J-C I=L^{(1)} \cdots L^{(m)} \tilde{L} U
$$

where $\tilde{L}$ is a lower triangular matrix (non bidiagonal, in general). However, if $\Delta_{s}^{(m)}=0$ for some $s \in\{0, \ldots, p-m-2\}$ then we can not assure the existence of a Darboux factorization (11) of $J-C I$ such that $\nu^{(m+1)}$ is defined as in (17).

\section{References}

[1] D. Barrios Rolanía, On the Darboux transform and the solutions of some integrable systems, Revista de la Real Academia de Ciencias Exactas, Fsicas y Naturales. Serie A. Matemticas 113 (2) (2019), pp. 13591378.

[2] D. Barrios Rolanía, A. Branquinho, Complex high order Toda lattices, J. Difference Equations and Applications, 15 (2) (2009), pp. 197-213.

[3] D. Barrios Rolanía, A. Branquinho, A. Foulquié Moreno, On the full Kostant-Toda system and the discrete Korteweg-de Vries equations, J. Math. Anal. Appl. 401 (2013), pp. 811-820.

[4] D. Barrios Rolanía, J.C. García-Ardila, Geronimus transformations for sequences of $d$-orthogonal polynomials, submitted (2019)

[5] D. Barrios Rolanía, J. S. Geronimo, G. López Lagomasino, High-order recurrence relations, Hermite-Padé approximation and Nikishin systems, Mat. Sb. 209 (3) (2018), 102137.

[6] D. Barrios Rolanía, D. Manrique, On the existence of Darboux transformations for banded matrices, Appl. Math. Comput. 253, 116-125 (2015)

[7] T. S. Chihara, An Introduction to Orthogonal Polynomials, Gordon and Breach Sc. Pub., Inc., New York, 1978.

[8] F. R. Gantmacher, The Theory of Matrices, Vol. I, AMS Chelsea Pub., Providence, Rhode Island, 2000.

[9] V. Kaliaguine, The operator moment problem, vector continued fractions and explicit form of the Favard theorem for vector orthogonal polynomials, J. Comput. Applied Math. 65, 181-193 (1995)

[10] P. Maroni, L'orthogonalit et les recurrences de polynômes d'ordre supérieur à deux, Annales de la Faculté des Sciences de Toulouse 5, Vol. 10 (1), 105-139 (1989)

[11] V. B. Matveev, Darboux transformation and the explicit solutions of differentialdifference and difference-difference evolution equations I, Lett. Math. Phys. 3 (3) (1979), pp. 217-222.

[12] V. B. Matveev, M.A. Salle, Differential-difference evolution equations II. Darboux transformation for the Toda lattice, Lett. Math. Phys. 3 (5) (1979), pp. 425-429. 
[13] J. Van Iseghem, Approximants de Padé vectoriales, These. Univ. des Sci et Tech. de Lille Flandre Artois, (1987) 